\title{
Disturbances of Rhythm Formation in Patients with Hemispheric Lesion
}

\author{
Hiroshi Nagasaki, Kenji Kosaka and Ryuichi NaKamura \\ Department of Rehabilitation Medicine, Tohoku University \\ School of Medicine, Narugo, Miyagi 989-68
}

\begin{abstract}
Nagasaki, H., Kosaka, K. and Nakamura, R. Disturbances of Rhythm Formation in Patients with Hemispheric Lesion. Tohoku J. exp. Med., 1981, 135 (3), 231-236 — A total of 191 patients with hemispheric lesion mainly due to CVD were examined by the finger-tapping test, and their disturbances of rhythm formation, 'hastening phenomenon' (HP), were compared with those of Parkinson's disease. HP was observed in $56 \%$ of the right and $49 \%$ of the left hemiplegic patients. HP in the intact hand of the patient was highly correlated with that of the affected hand. CT scans were examined in 86 of 191 patients, and HP was specifically correlated with unilateral striatal lesions. Thus, the disturbances of rhythm formation in hemiplegia, together with those in Parkinson's disease, Huntington's chorea, SND and OPCA, were attributed either to organic or to functional deficits in the striatum. disturbances of rhythm formation; hemiplegia; finger-tapping test; striatal lesion
\end{abstract}

Rhythmic movements are often disturbed characteristically in patients with Parkinson's disease; e.g., festination or freezing in gait or speech. In the finger-tapping test in which the patient is instructed to respond synchronously to a periodic sound signal by finger-tap, this disturbance is typically observed; synchronized responses fail at a critical frequency $\left(f_{t}\right)$, and a desynchronized response with a hastened rate $\left(f_{h}=5 \sim 6 \mathrm{~Hz}\right)$ appears at the frequencies higher than $f_{t} ; f_{t}$ and $f_{k}$ are constant in each of the patients. This disturbance is called 'hastening phenomenon' (HP; Nakamura et al. 1978a). HP is considered to represent quantitatively the disturbances of rhythm formation. Being disturbed by the unwilling release of the abnormal rhythm with a hastened rate, the patients are unable to form an expected rhythm exactly and steadily. Motor performances of the patients with HP are more impaired than those without HP (Nakamura et al. 1976). According to the statistical analysis (Nakamura et al. 1978b), the disturbance of the rhythm formation (HP) is considered one of clinical features in Parkinson's disease which is independent of rigidity or tremor and closely related to akinesia. HP was seen in all the patients with 'pure akinesia' who had neither rigidity nor tremor.

Apart from Parkinson's disease, HP is frequently observed in several diseases of the central nervous system, namely, Huntington's chorea, strio-nigral degeneration (SND), olivo-ponto-cerebellar atrophy (OPCA), normo-pressure

Received for publication, December 12, 1980. 
hydrocephalus (NPH) and hemiplegia due to cerebrovascular disease (CVD). On the contrary, lesions confined to the thalamus and cerebellum rarely result in HP. These findings imply that the disturbances of the rhythm formation are prevailing in a variety of neurological disorders and that they are closely related to the specific lesions in the brain.

In the present study, patients with hemiplegia were examined by the fingertapping test and HP was found to be correlated with the site and extent of unilateral hemispheric lesions.

\section{Methods}

\section{Subject}

The finger-tapping test was examined in 101 patients with right hemiplegia and 90 with left hemiplegia. Most cases were due to CVD except a few with head injuries or intracranial tumors. Those who suffered from the disease within the last three months were excluded.

\section{Finger-tapping test}

Periodic signals of $1,2,2.5,3,3.5,4,4.5$ and $5 \mathrm{~Hz}$ were given successively in the order. In each of the signals about 50 trials of tapping response were performed. Detailed procedure of the test was described elsewhere (Nakamura et al. 1978a). Not only the intact hand but the affected one, if possible, was examined.

\section{Computerised tomography $(C T)$}

CT scans were examined in 86 of 191 patients subjected to the finger-tapping test. Most of the scans were taken within 3 months after the finger-tapping test. For each patient 8 separate slices were given in the usual operation plane of the scanner, that is, approximately 10-15 degrees to the horizontal plane. Low-density area in slice was traced on a corresponding anatomical map.

\section{Results and Discussion}

\section{Hastening phenomenon (HP)}

Response relations, i.e., signal frequency vs. mean response frequency, were studied by the finger-tapping test. HP of the patients with hemiplegia was often different in pattern from that of patients with Parkinson's disease. In consequence, responses specific to hemiplegia were classified into four types from 0 to III (Fig. 1). Type 0 represented a normal (synchronized) response up to $5 \mathrm{~Hz}$, namely $\mathrm{HP}(-)$, whereas HP was observed in types I-III, i.e., $\mathrm{HP}(+)$. In type III, a pattern of HP similar to that of Parkinson's disease was seen; the response became fast abruptly at $f_{t}$ and its frequency was kept nearly constant throughout the range of the signal frequencies higher than $f_{t}$. In type I, response frequency was faster than the signal frequency, but the difference was rather small, within $0.5 \mathrm{~Hz}$. Although small, this discrepancy is significant since in normal subjects the response synchronized with the signal within range of $\pm 0.01 \mathrm{~Hz}$. Type II had a pattern of the response intermediate between types I and III; the deviation of the response frequency from the signal gradually became greater than $0.5 \mathrm{~Hz}$. 

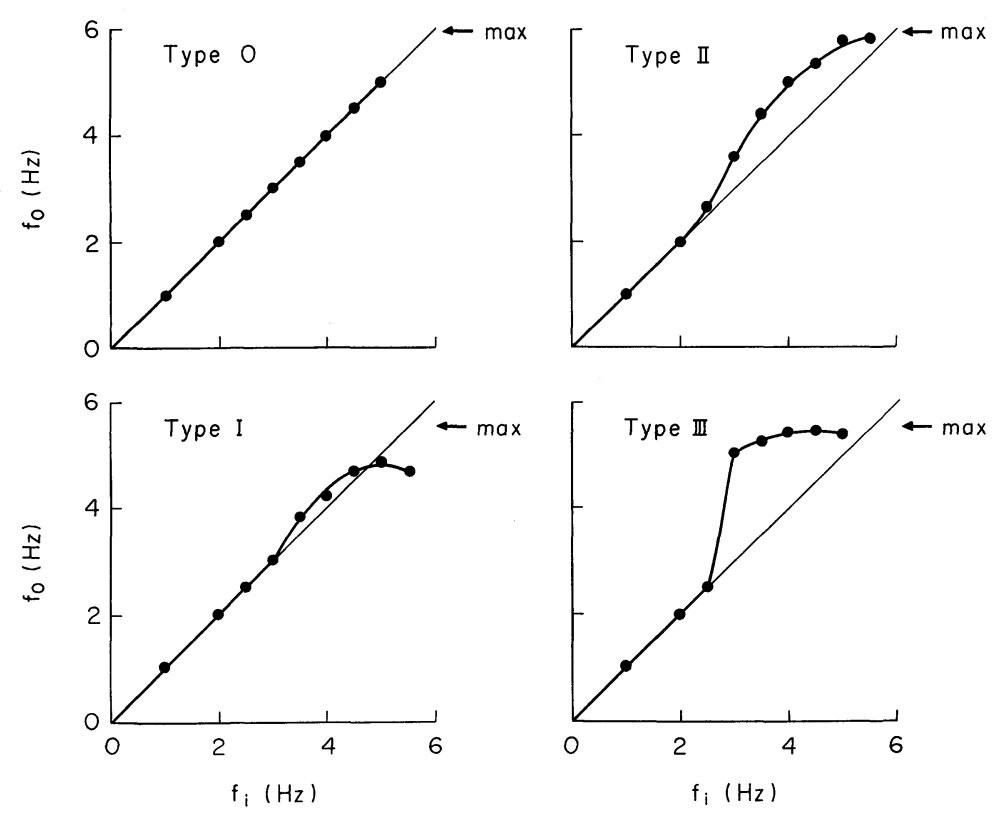

Fig. 1. Four types of the tapping response in hemiplegia. $f_{i}$, signal frequency ; $f_{0}$, mean response frequency of finger-tapping; max, maximum frequency of finger tapping.

TABLE 1. Correlation of the pattern of the tapping response for the intact hand with that for the affected hand

\begin{tabular}{cccccc}
\hline \multirow{2}{*}{$\begin{array}{c}\text { Left } \\
\text { hand }\end{array}$} & \multicolumn{4}{c}{ Right hand } \\
\cline { 2 - 3 } & \multicolumn{2}{c}{ Right hemiplegia } & & \multicolumn{2}{c}{ Left hemiplegia } \\
\cline { 2 - 3 } \cline { 5 - 6 } & HP (-) & HP (+) & & $H P(-)$ & $H P(+)$ \\
\hline HP (-) & 11 & 2 & & 20 & 3 \\
HP (+) & 8 & 10 & & 0 & 7 \\
\hline
\end{tabular}

HP (-): type 0. HP (+): types I-III.

In the patients subjected to the test with both hands, performances of the two hands were compared with each other. The pattern of response for the intact hand was highly correlated with that for the affected hand (Table 1). There were only two patients whose affected hand showed HP but the intact hand did not. These results indicate that the disturbances of the rhythm formation influence bilaterally the patient's movements, and that it is possible to detect the disturbances through the examination of only the intact hand. In Table 1, it is also noticed that the intact hand often shows HP although the affected hand gives a normal response up to $4 \mathrm{~Hz}$. Due to the deficits of motor function, the paralyzed hand could not perform the tapping with abnormally high rate.

According to the classification described above, the results of the finger-tapping test in 191 patients are given in Table 2. Subjects with HP at least in one hand 
TABLE 2. Results of the finger-tapping test for 191 patients

\begin{tabular}{|c|c|c|c|c|c|c|}
\hline & \multicolumn{4}{|c|}{ Type } & \multirow{2}{*}{ Total } & \multirow{2}{*}{$\mathrm{HP}(+) \%$} \\
\hline & 0 & $I$ & II & III & & \\
\hline Right hemiplegia & 44 & 16 & 26 & 15 & 101 & 56 \\
\hline Left hemiplegia & 46 & 13 & 21 & 10 & 90 & 49 \\
\hline Total & 90 & 29 & 47 & 25 & 191 & 53 \\
\hline
\end{tabular}

Type 0: HP (-). Types I-III: HP (+).
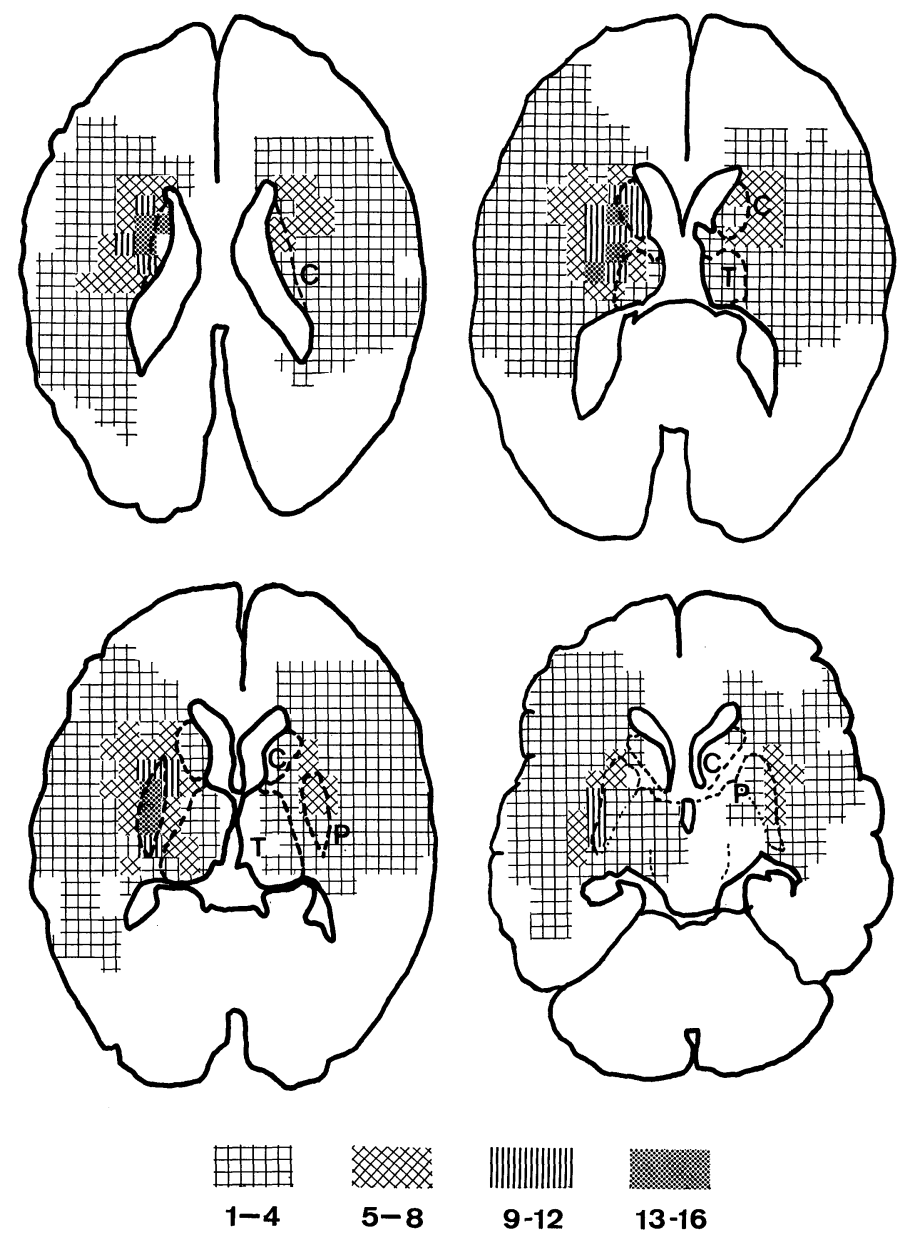

Fig. 2. Overlapped distribution of the unilateral hemispheric lesions for 29 right and 13 left hemiplegic patients with $\mathrm{HP}(+)$. C, caudate; $\mathrm{P}$, putamen; $\mathrm{T}$, thalamus.

were regarded as $\mathrm{HP}(+)$. When the type was different between both hands, it was represented by a type of higher class. It is noticed that the occurrence of HP is more frequent in the right hemiplegic patients $(56 \%)$ than the left $(49 \%)$ and 
that type II, not type III as in Parkinson's disease, is representative of the patterns of HP for the hemiplegic patients (Table 2).

\section{$H P$ and localization of brain lesion}

In 17 of 86 patients examined, lesions were recognized only in the cerebral cortical area. Four of these patients with lesions not extending to the frontal lobe gave a normal tapping response, $\mathrm{HP}(-)$. On the contrary, 10 out of the other 13 patients with frontal lesions were $\mathrm{HP}(+)$. This suggests that $\mathrm{HP}$ is related especially to frontal lesions.

Apart from these 17 patients with cortical lesions, all of the other 69 patients had either unilateral subcortical lesions or no lesions. In Fig. 2, for $42 \mathrm{HP}(+)$ patients with unilateral subcortical lesions, low-density area of the CT scan was superimposed on each of several planes of the scan. Fig. 2 demonstrates clearly that in both hemispheres the distribution of the lesions concentrates in the striatum (caudate and putamen) but not in the thalamus. This is shown in detail in Table 3 where the types of the tapping response are related to the site and extent of the lesion in 69 patients. According to Table 3, type 0, which means a normal response, is related either to thalamic lesions (11 of 27) or to no lesions (10 of 27). Only $20 \%$ of the patients in this type have lesions including the striatum. On the contrary, HP is highly correlated with striatal lesions; more than $90 \%$ of types I, II and III have lesions in the caudate and/or the putamen. Especially in type III, which is similar to that of Parkinson's disease, all patients examined have striatal lesions. It seems that the damage in the putamen is essential to the typical hastening phenomenon.

In conclusion, the disturbances of rhythm formation in hemiplegic patients are specifically correlated with striatal lesions. On the other hand, in Parkinson's disease, in which $72 \%$ of the patients are $\mathrm{HP}(+)$, a functional deficit of the striatum is caused by degenerative changes of substantia nigra. Pathological changes of the striatum are also common to Huntington's chorea, SND and OPCA, in which every patient hitherto examined was $\mathrm{HP}(+)$. Consequently, these

TABLE 3. Correlation of the types of the tapping response with the site and the extent of unilateral subcortical lesion in 69 patients

\begin{tabular}{|c|c|c|c|c|c|c|c|c|}
\hline \multirow[b]{3}{*}{ Type } & \multirow[b]{3}{*}{$\begin{array}{l}\text { Number } \\
\text { of cases }\end{array}$} & \multicolumn{7}{|c|}{ Lesion } \\
\hline & & \multicolumn{4}{|c|}{ Striatum } & \multirow{2}{*}{$\begin{array}{l}\text { Localized } \\
\text { in thalamus } \\
\text { and internal } \\
\text { capsule }\end{array}$} & \multirow{2}{*}{$\begin{array}{c}\text { Dilatation } \\
\text { of } \\
\text { ventricle }\end{array}$} & \multirow[t]{2}{*}{ No lesion } \\
\hline & & Caudate & $\begin{array}{l}\text { Caudate } \\
\text { and } \\
\text { putamen }\end{array}$ & Putamen & Case (\%) & & & \\
\hline 0 & 27 & 2 & 4 & 0 & 20 & 11 & 2 & 10 \\
\hline I & 13 & 2 & 7 & 3 & 92 & 1 & 1 & 0 \\
\hline II & 21 & 8 & 8 & 4 & 95 & 1 & 7 & 0 \\
\hline III & 8 & 0 & 6 & 2 & 100 & 0 & 3 & 0 \\
\hline \multicolumn{9}{|l|}{ Sum of } \\
\hline I-III & 42 & 10 & 21 & 9 & 95 & 2 & 11 & 0 \\
\hline
\end{tabular}


facts imply that the disturbances of rhythm formation (HP) are to be attributed either to organic or to functional deficits in the striatum.

\section{Acknowledgments}

We express our thanks to Dr. H. Narabayashi of Juntendo University, Dr. M. Mizukami of Mihara Memorial Hospital, Dr. Y. Nakazawa of Yamanashi Onsen Hospital and Dr. T. Amakusa of Amakusa Hospital for their courtesy in making this study in their departments.

\section{References}

1) Nakamura, R., Nagasaki, H. \& Narabayashi, H. (1976) Arrhythmokinesia in parkinsonism. In: Advance in Parkinsonism. edited by W. Birkmayer \& 0 . Hornykiewicz, Editiones Roche, Basle, pp. 258-268.

2) Nakamura, R., Nagasaki, H. \& Narabayashi, H. (1978a) Disturbances of rhythm formation in patients with Parkinson's disease: Part I, Characteristics of tapping response to the periodic signals. Percept. Motor Skills, 46, 63-75.

3) Nakamura, R., Taniguchi, R., Nagasaki, H. \& Imai, T. (1978b) Statistical analysis of finger-tapping test for 123 patients with Parkinson's disease. Annual Reports of Special Research Project of the Etiopathogenesis of "Nambyo", 599-605. (Japanese) 